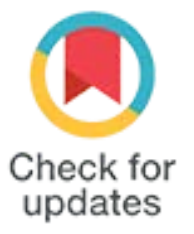

DOI: $10.29252 /$ ijcp-25262

\section{Prevalence of Coronary Artery Disease and Its Risk Factors in Patients Undergoing Permanent Pacemaker Implantation}

\author{
Ashwal Jayaram ' , Jerry Iype ${ }^{2}$, Deeksha Karkera ${ }^{2}$, M Sudhakar \\ Rao ${ }^{1, *}$ (D), Tom Devasia 1, Padmakumar Ramachandran 1, \\ Umesh Pai $^{2}$, Jyothi Samanth ${ }^{2}$, Ganesh Paramasivam ${ }^{1}$
}

${ }^{1}$ Department of Cardiology, Kasturba Medical College, Manipal Academy of Higher Education, Manipal

${ }^{2}$ Department of Cardiovascular Technology, School of Allied Health Science (SOAHS), Manipal Academy of Higher Education, Manipal

* Corresponding author: Sudhakar Rao, Assistant Professor, Department of Cardiology, Kasturba Medical College, Manipal Academy of Higher Education, Manipal -576104, India. Tel: +91-9902547712, E-mail: msudhakar88@gmail.com

\begin{tabular}{l} 
Submitted: 27-04-2019 \\
Accepted: 25-06-2019 \\
\hline Keywords: \\
Tachycardia, Sinoatrial \\
Nodal Reentry \\
Sick Sinus Syndrome \\
Atrioventricular Node \\
Congenital heart block \\
Coronary Artery Disease \\
Pacemaker, Artificial \\
\hline C) 2019. International Journal \\
of Cardiovascular Practice.
\end{tabular}

\section{INTRODUCTION}

Symptomatic bradyarrhythmias occur in older patients who have multiple coronary risk factors and often present with angina-like symptoms [1]. Bradycardia is a common clinical phenomenon and can be defined as heart rate below $60 \mathrm{bpm}$. It may also be associated with various rhythmic disorders such as sinus nodal disease (SND) and atrioventricular (AV) blocks [2]. Patients with regular or occasional conduction disturbance who experience symptoms such as fatigue, presyncope or syncope require permanent pacemaker implantation
(PPI) [3]. Many studies have shown that conduction disturbances may be associated with acute conditions such as myocardial infarction (MI), electrolytic imbalance, certain drugs, myocardial disease, cardiac injury and chronic conditions such as degenerative diseases, cardiomyopathies, hypertension and congenital heart disease (CHD) [3]. Some pacemaker implanted patients also have a dormant atherosclerotic disease which promotes conduction system ischemia and produces conduction disturbances [3]. This study 
was performed to assess the prevalence of $\mathrm{CAD}$ and its risk factors in patients undergoing permanent pacemaker implantation.

\section{MATERIALS AND METHODS}

\section{Study Design \& Patient Population}

This prospective, single-center hospital based study was conducted at a tertiary-care center in India between 2011 and 2015. Consecutive patients older than 40 years requiring PPI were enrolled. Patients who presented with symptomatic sick sinus syndrome (SSS) or conduction disorders and fulfilled ACCF/AHA/HRS criteria for PPI [4], were considered for enrollment. Exclusion criteria were as follows; (a) acute coronary syndrome (ACS); (b) myocarditis; (c) infective endocarditis; (d) sepsis; (e) connective tissue disorders; (f) infiltrative disorders; (g) muscle dystrophies or systemic malignancies; (h) CHD, valvular heart disease and cardiomyopathy; (i) endstage renal disease or electrolytic disturbances; $(j)$ patients taking drugs such as digoxin, beta-blockers, calcium channel blockers or anti-arrhythmic agents; (k) patients with allergies to contrast agents; and (1) patients who did not consent for the study.

An informed consent was obtained from each patient. The study was conducted in compliance with the Declaration of Helsinki. The study protocol was approved by the Institutional Ethics Committee.

\section{Data Collection}

A detailed history of presenting symptoms, past history of similar complaints, family history of $\mathrm{CAD}$ or conduction disorders, presence of atherosclerotic risk factors and drug intake were collected from all patients. Complete physical examination and cardiovascular examinations were performed. Investigations such as electrocardiogram (ECG), complete blood count, kidney and liver function tests, lipid profile, fasting blood sugar, thyroid profile along with serum electrolytes were obtained. Echocardiography was performed in all cases to rule out structural heart disease or cardiomyopathies as well as to document left ventricular function.

\section{Coronary Angiography}

Coronary angiography (CAG) was performed in all patients by the standard Judkins technique. The angiograms were assessed by two independent cardiologists. $\mathrm{CAD}$ was defined as $50 \%$ diameter stenosis [visual estimation as well as quantitative coronary analysis (QCA) of lesion diameter, comparing it with the adjacent normal segment] in at least one major coronary artery [left anterior descending (LAD), left circumflex (LCX) or right coronary artery (RCA)]. $\mathrm{CAD}$ was considered as single vessel disease (SVD), double vessel disease (DVD) or triple vessel disease (TVD) according to the number of major arteries or their branches with obstructive (> $50 \%$ diameter stenosis) atherosclerotic involvement.

\section{Statistical Analysis}

All continuous variables were expressed as mean value \pm standard deviation and categorical variables were expressed as frequency and percentages. Comparisons were performed for two groups, patients with pacemaker in those with $\mathrm{CAD}$ or without $\mathrm{CAD}$. Group comparisons were performed by Student t-test for continuous variables. Chi-square test or Fisher exact test was used for categorical variables. Odds ratio and 95\% confidence interval of individual CAD risk factors were calculated. A p-value of $<0.05$ was considered statistically significant. Statistical analysis was performed by Statistical Package for Social Sciences (IBM SPSS; Chicago, IL, USA) program, version 20.

\section{RESULTS}

\section{CAD Prevalence and Prognosis}

During the study period, 258 patients underwent PPI. Of them, 137 (53.1\%) were males and 121 (46.9\%) females. $62.0 \%$ of patients (160 of 258 patients) had complete heart block, $25.2 \%$ (65 of 258 patients) had sick sinus syndrome (SSS), 9.3\% (24 of 258 patients) had symptomatic sinus bradycardia and $3.5 \%$ ( 9 of 258 patients) had AV/infrahisian block. Overall prevalence of CAD in patients who underwent PPI was 19.37\% (50 of 258 patients), as $10.46 \%$ (27 of 258 patients), $6.20 \%$ (16 of 258 patients) and $2.71 \%$ (7 of 258 patients) patients exhibited single vessel disease, double vessel disease and triple vessel disease, respectively. LAD, RCA and LCX obstruction was observed in $12.0 \%, 11.2 \%$ and $7.7 \%$ patients, respectively. In total, $1.55 \%$ of patients underwent bypass surgery and $4.26 \%$ patients had past history of CAD.

\section{Univariate Analysis of CAD Risk Factors}

The univariate analysis of demographic characteristics in presence of CAD is detailed in Table 1. Mean age of patients without $\mathrm{CAD}$ was $62.1 \pm 14.64$ years and in those with $\mathrm{CAD}$ was $69.2 \pm 9.14(\mathrm{P}=0.001)$. Among 50 patients who had $\mathrm{CAD}, 52.5 \%$ were smokers $(\mathrm{P}=$ $0.008), 50.0 \%$ had diabetes $(\mathrm{P}<0.0001), 64.0 \%$ had hypertension $(\mathrm{P}=0.004), \quad 32.0 \%$ had hypercholesterolemia $(\mathrm{P}=0.05), 36.0 \%$ had alcohol addiction $(\mathrm{P}=0.5)$ and $8.0 \%$ had $\mathrm{CKD}(\mathrm{P}=0.7)$. Age of $\geq 76.5$ years had sensitivity of $12.1 \%$ and specificity of $78.8 \%$ for detection of $\mathrm{CAD}$ as depicted in Figure 1 with Receiver operating characteristic (ROC) curve.

\section{Multivariate Analysis of CAD Risk Factors}

Multivariate analysis of age, gender, diabetes, smoking, hypertension and hypercholesterolemia was performed. The analysis showed that age (odds ratio: 1.042; 95\% confidence interval: 1.009-1.075; $\mathrm{P}=0.01$ ) and diabetes (odds ratio: 3.437 ; $95 \%$ confidence interval: 1.618-7.303; $\mathrm{P}=0.001$ ) had a statistically significant association with $\mathrm{CAD}$. The multivariate analysis of the demographic characteristics in presence of $\mathrm{CAD}$ is shown in Table 2. 
Table 1. Univariate Analysis of Age, Gender, Smoking, Diabetes, Hypertension, Alcohol Intake and Chronic Kidney Disease with the Presence of Coronary Artery Disease

\begin{tabular}{lccc}
\hline & Coronary Artery Disease & Pbent $(\mathbf{N = 2 0 8})$ & Present $(\mathbf{N}=\mathbf{5 0})$ \\
\hline Age (years) & $62.10 \pm 14.635$ & $69.20 \pm 9.138$ & 0.001 \\
\hline Age & & & 0.03 \\
$\quad<45$ years & $25(12.0)$ & $0(0)$ & \\
$\quad 45-60$ years & $64(31.3)$ & $8(16)$ & \\
$\quad>60$ years & $118(56.7)$ & $42(84)$ & \\
Gender & & & \\
$\quad$ Male & $103(49.5)$ & $34(68.0)$ & \\
$\quad$ Female & $105(50.5)$ & $16(32.0)$ & \\
Smoking & $65(31.3)$ & $26(52.0)$ & \\
Diabetes & $41(19.7)$ & $25(50.0)$ & \\
Hypertension & $85(40.9)$ & $32(64.0)$ & 0.02 \\
Hypercholesterolemia & $39(18.8)$ & $16(32.0)$ & 0.0001 \\
Alcohol Intake & $64(30.8)$ & $18(36.0)$ & 0.004 \\
CKD & $14(6.7)$ & $4(8.0)$ & 0.05 \\
\hline
\end{tabular}

Data in table are presented as No. (\%) or Mean \pm SD

CKD: chronic kidney disease

Table 2. Multivariate Analysis of Diabetes, Smoking, Hypertension, Hypercholesterolemia, Gender and Age with the Presence of Coronary Artery Disease by Logistic Regression

\begin{tabular}{|c|c|c|c|c|}
\hline & \multirow[t]{2}{*}{ Odds ratio } & \multicolumn{2}{|c|}{ 95\% Confidence Interval } & \multirow[t]{2}{*}{ P Value } \\
\hline & & Lower & Upper & \\
\hline Diabetes & 3.437 & 1.618 & 7.303 & 0.001 \\
\hline Smoking & 1.964 & .785 & 4.916 & 0.1 \\
\hline Hypertension & 1.656 & 0.799 & 3.431 & 0.1 \\
\hline Hypercholesterolemia & 1.115 & 0.487 & 2.554 & 0.7 \\
\hline Female & 0.712 & 0.276 & 1.837 & 0.4 \\
\hline Age & 1.042 & 1.009 & 1.075 & 0.01 \\
\hline
\end{tabular}

Table 3. Univariate Analysis of Indication of Pacemaker with Involvement of Individual Coronary Arteries.

\begin{tabular}{lccc}
\hline & Sinus nodal disease, $(\mathbf{n}=\mathbf{8 9})$ & AV Nodal/Infra-Hisian Disease, $(\mathbf{n}=\mathbf{1 6 9})$ & P Value \\
\hline Normal coronaries & $80(89.9)$ & $128(75.7)$ & 0.004 \\
Abnormal coronaries & $9(10.1)$ & $41(24.3)$ \\
LAD & $3(3.4)$ & $28(16.6)$ & $22(13.0)$ \\
RCA & $7(7.8)$ & $15(8.9)$ & 0.01 \\
LCX & $5(5.6)$ & 0.2 \\
\hline
\end{tabular}

Data in table are presented as No. (\%)

LAD: Left anterior descending coronary artery; RCA: right coronary artery; LCX: left circumflex coronary artery; AV: atrioven tricular

\section{Indication of Pacemaker with Involvement of Individual Coronary Arteries}

Indications were grouped into those affecting sinus node like SSS along with sinus bradycardia and those affecting AV node like varying degrees of AV nodal/complete heart block or infra-hisian blocks and with involvement with individual arteries. Among 258 patients, 89 patients $(34.5 \%)$ had involvement of sinus node whereas 169 patients $(65.5 \%)$ had involvement of AV node. Of those 89 patients with involvement of sinus node, 65 patients ( $73 \%)$ had SSS and $24(27 \%)$ had symptomatic bradycardia. In 169 patients with involvement of AV node, 160 patients (95\%) had complete heart block and $9(5 \%)$ had varying degrees of AV nodal blocks/infra-hisian blocks. Among 89 patients with sinus node disease, 3 (3.4\%) had LAD lesions, 7 (7.8\%) had RCA lesions and 5 (5.6\%) had LCX lesions. Among 169 patients with AV node disease, 28 (16.6\%) had LAD lesions, 22 (13.0\%) RCA lesions and 15 (8.9 $\%)$ LCX lesions. The univariate analysis of indication of pacemaker with involvement of individual coronary artery is described in Table 3.



Figure 1. Receiver operating characteristic (ROC) curve to identify the best cut-off value of vitamin $\mathrm{D}$ in predicting the presence of coronary artery disease. In patients undergoing permanent pacemaker implantation, patients with age $\geq 76.5$ years had sensitivity of $12.1 \%$ and specificity of $78.8 \%$ in detection of a coronary artery disease 


\section{DISCUSSION}

Our study found overall prevalence of CAD in patients older than 40 years who underwent PPI as $19.4 \%$ (50/258 patients). Very limited data was obtained on prevalence of $\mathrm{CAD}$ and $\mathrm{CAD}$ risk factors amongst patients undergoing a permanent pacemaker. Our study showed that CHB or 3rd degree AV block (62\%) and SSS (25.2\%) were the two main indications for implantation of a pacemaker.

Prevalence of CAD in chronic conduction disturbance has been reported as $15-70 \%$ depending on patient characteristics, diagnostic modality and criteria used to define $\mathrm{CAD}[1,5-7]$. In a necropsy series of 100 patients with chronic heart block, Davies [5] reported that only $15 \%$ of patients had $\mathrm{CAD}$ of sufficient severity to account for heart block. During 1960 CAD prevalence rates were based on autopsy reports and insurance statistics. According to these studies, prevalence rates varied between $0.49-0.63 \%$ [8].

Hseuh et al. [1] performed selective CAG on 113 patients with symptomatic bradyarrhythmia and found incident CAD in $20 \%$ of these patients. Brueck et al. [6] in an angiographic study of 507 patients requiring pacemaker implantation and at least one atherosclerotic risk factor, found a remarkable $71 \%$ incidence of $C A D$. Alai et al. [2] revealed an overall $45 \%$ incidence of $\mathrm{CAD}$ and $29 \%$ incidence of significant $\mathrm{CAD}$ in these patients. Our study revealed a prevalence rate of $19.4 \%$ of CAD in patients who underwent PPI during 2011-2015.

Our study also showed that males $(68.0 \%)$ were more affected than females (32.0\%) and the elderly group (> 60 years) was more prone to $\mathrm{CAD}$. Our study also showed that coronary angiography in patients older or equal 76.5 years had sensitivity of $12.1 \%$ and specificity of $78.8 \%$ in detection of a CAD. Hseuh et al. [1] in a study included 113 patients of whom $68(60.2 \%)$ were males and $45(39.8 \%)$ females. In another study, prevalence of $\mathrm{CAD}$ was 6.5 times higher in males than females [8].

Transient AV block is a common complication of acute MI [especially inferior wall myocardial infarction (IWMI)]. Chronic CAD is one of the causes of AV block although it is a much less common cause than idiopathic fibrosis of the conduction system [9]. Ginks et al. [10] showed that among 30 patients who were enrolled for PPI without any symptoms of CAD, CAG revealed $\mathrm{CAD}$ in 13 of the patients (43\%).

Bassan et al. [11] in a prospective study showed that 11 of $51(21.6 \%)$ patients who survived an IWMI had some degree of AV block and $90 \%$ of patients with AV block had simultaneous obstruction of RCA and LAD. Patients with IWMI and LAD artery obstruction had a six fold higher risk for AV block if the LAD had $75 \%$ or more stenosis before the second septal perforator. The presence of heart block had a sensitivity, specificity and predictive value of $31 \%, 95 \%$, and $91 \%$ for the presence of $\mathrm{LAD}$ obstruction, respectively. However, these findings of Bassan et al. are contradicted by Thrombolysis in Acute Myocardial Infarction (TAMI) [12] and Thrombolysis in Myocardial Infarction (TIMI) [13] populations. Many studies have proven that the LAD is the most common coronary artery involved by atherosclerosis, especially type $\mathrm{V}$ atherosclerosis and in myocardial infarction [14-16]. Our study showed that $12.0 \%, 11.2 \%$ and $7.7 \%$ of patients had significant LAD, RCA and LCX obstruction, respectively.

In our study, LAD stenosis was associated with the presence of AV nodal/infra-hisian disease when compared to sinus nodal disease (SND) as shown in Table 3. RCA and LCX were not statistically associated with either SND or the AV nodal/infra-hisian disease. In acute MI especially IWMI, RCA stenosis is associated with AV nodal/infra-hisian dysfunction in the form of varying degrees of AV block. The patients with ACS requiring pacemaker were excluded in our study group and all had presumed chronic degenerative etiology. Thus, we can conclude that compared to patient with sinus nodal disease, LAD lesion was more prevalent in AV nodal disease patients.

Mortality in patients with SND is determined by nature and progression of associated cardiac or systemic disease, control of tachyarrhythmia and risk of thromboembolic complications. In a study of 148 patients who received pacemaker for SND, the survival rate at 1,5 and 10 years were $84 \%, 57 \%$ and $27 \%$, respectively which showed decreased life expectancy [9].

The SND trial which included 2010 elderly patients showed that most deaths were due to non-cardiac causes [9]. Patients who present with acute MI and intraventricular conduction defects with the exception of having left anterior hemi-block have an unfavorable prognosis and a higher incidence of sudden cardiac death. It is associated with extensive myocardial necrosis, malignant arrhythmias and pump failure [9]. It has been indicated that coronary revascularization (both percutaneous coronary intervention and bypass grafting) has little if any, role in reversing the conduction defect in case of chronic degenerative conduction blocks. Therefore, we proceeded with pacemaker insertion in these patients and they were re-vascularized later. Appropriate management of $\mathrm{CAD}$ with pharmacological or interventional therapy is also more likely to improve the long-term outlook of these patients.

\section{Limitations}

Though we included 258 patients for pacemaker insertion, none of them were subjected to electrophysiological (EP) study to identify the exact location of conduction block. This was mainly because in routine practice as per ACC/AHA guidelines, we usually offer pacemaker depending on clinical 
presentation and very rarely perform EP studies to prove the need for pacemaker insertion.

\section{CONCLUSION}

CAD was present in $19.4 \%$ of patients undergoing pacemaker implantation signifying a strong association between conduction blocks and CAD. LAD stenosis is significantly associated with AV nodal/infra-hisian disease. Age and diabetes have a strong association with the presence of $\mathrm{CAD}$ implying the fact that people older than 70 years and with diabetes should be screened for CAD.

\section{Conflict of Interest}

There is no conflict of interests with this article.

\section{Funding/Supports}

This research did not receive any specific grant from funding agencies in the public, commercial or not-forprofit sectors.

\section{REFERENCES}

1. Hsueh CW, Lee WL, Chen YT, Ting CT. The incidence of coronary artery disease in patients with symptomatic bradyarrhythmias. Jpn Heart J. 2001;42(4):417-23. doi: 10.1536/jhj.42.417 pmid: 11693278

2. Alai MS, Beig JR, Kumar S, Yaqoob I, Hafeez I, Lone AA, et al. Prevalence and characterization of coronary artery disease in patients with symptomatic bradyarrhythmias requiring pacemaker implantation. Indian Heart J. 2016;68 Suppl 3(Suppl II):S21-S5. doi: 10.1016/j.ihj.2016.06.013 pmid: 28038720

3. Mosseri M, Izak T, Rosenheck S, Lotan C, Rozenman Y, Zolti $\mathrm{E}$, et al. Coronary angiographic characteristics of patients with permanent artificial pacemakers. Circulation. 1997;96(3):80915. doi: 10.1161/01.cir. 96.3 .809 pmid: 9264486

4. Epstein $\mathrm{AE}, \mathrm{DiMarco} J \mathrm{~J}$, Ellenbogen KA, Estes NA, 3rd, Freedman RA, Gettes LS, et al. 2012 ACCF/AHA/HRS focused update incorporated into the ACCF/AHA/HRS 2008 guidelines for device-based therapy of cardiac rhythm abnormalities: a report of the American College of Cardiology Foundation/American Heart Association Task Force on Practice Guidelines and the Heart Rhythm Society. J Am Coll Cardiol. 2013;61(3):e6-75. doi: 10.1016/j.jacc.2012.11.007 pmid: 23265327

5. Davies M, Path M. Pathology of conducting tissue of the heart. London: Butterworth; 1971.
6. Brueck M, Bandorski D, Kramer W. Incidence of coronary artery disease and necessity of revascularization in symptomatic patients requiring permanent pacemaker implantation. Med Klin (Munich). 2008;103(12):827-30. doi: 10.1007/s00063-008-1130-z pmid: 19099211

7. Ciaroni S, Bloch A, Albrecht L, Vanautryve B. Diagnosis of coronary artery disease in patients with permanent cardiac pacemaker by dobutamine stress echocardiography or exercise thallium-201 myocardial tomography. Echocardiography. 2000;17(7):675-9. doi: 10.1046/j.1540-8175.2000.00675.x pmid: 11107204

8. Sriharibabu M. Changing trends in the prevalence of coronary heart disease. Indian Heart J. 2016;68(4):445-6. doi: 10.1016/j.ihj.2016.04.008 pmid: 27543464

9. Crawford MH, DiMarco JP, Paulus WJ. Cardiology. 3rd ed. Philadelphia, USA: Elsevier; 2010.

10. Ginks W, Sutton R, Siddons H, Leatham A. Unsuspected coronary artery disease as cause of chronic atrioventricular block in middle age. Br Heart J. 1980;44(6):699-702. doi: 10.1136/hrt.44.6.699 pmid: 6970044

11. Bassan R, Maia IG, Bozza A, Amino JG, Santos M. Atrioventricular block in acute inferior wall myocardial infarction: harbinger of associated obstruction of the left anterior descending coronary artery. J Am Coll Cardiol. 1986;8(4):773-8. doi: 10.1016/s0735-1097(86)80416-8 pmid: 3760353

12. Clemmensen P, Bates ER, Califf RM, Hlatky MA, Aronson L, George BS, et al. Complete atrioventricular block complicating inferior wall acute myocardial infarction treated with reperfusion therapy. TAMI Study Group. Am J Cardiol. 1991;67(4):225-30. doi: 10.1016/0002-9149(91)90550-5 pmid: 1899319

13. Berger PB, Ruocco NA, Jr., Ryan TJ, Frederick MM, Jacobs $\mathrm{AK}$, Faxon DP. Incidence and prognostic implications of heart block complicating inferior myocardial infarction treated with thrombolytic therapy: results from TIMI II.J Am Coll Cardiol. 1992;20(3):533-40. doi: 10.1016/0735-1097(92)90004-7 pmid: 1512330

14. Jayaram AA, Shah S. Risk factors, clinical features, angiographic characteristics and treatment outcomes of young myocardial infarction patients. J Indian College Cardio. 2015;5(3):203-8. doi: 10.1016/j.jicc.2015.05.002

15. Jha B, Naik D, Agarwal A, Jana S, Patel M. Incidence of atherosclerosis in different coronary arteries and its relation with myocardial infarction- A randomized study in 300 autopsy hearts in a tertiary care hospital. Int J Med Sci Public Health. 2013;2(4):836. doi: 10.5455/ijmsph.2013.200620131

16. Beig JR, Shah TR, Hafeez I, Dar MI, Rather HA, Tramboo NA, et al. Clinico-angiographic profile and procedural outcomes in patients undergoing percutaneous coronary interventions: The Srinagar registry. Indian Heart J. 2017;69(5):589-96. doi: 10.1016/j.ihj.2017.01.021 pmid: 29054181 